\title{
A Dynamic Spectrum Access Scheme for Cognitive Radio Networks
}

\author{
K. -L. Du, M. N. S. Swamy \\ Department of Electrical and Computer Engineering \\ Concordia University \\ Montreal, Canada, H3G 1M8 \\ E-mail: kldu@ieee.org, swamy@ece.concordia.ca
}

\author{
Qiang Ni \\ Electronics and Computer Engineering Division \\ School of Engineering and Design, Brunel University \\ London, United Kingdom, UB8 3PH \\ Email: qiang.ni@brunel.ac.uk
}

\begin{abstract}
In this paper, the dynamic spectrum access problem for cognitive radio (CR) networks is formulated as maximizing the sum channel capacity while satisfying the power budgets of individual secondary user radios as well as the SINR constraints on both the secondary and primary users. By applying the Karush-Kuhn-Tucker theorem, we derive a waterfilling soluton. An iterative water-filling algorithm is proposed for implementing joint channel and power allocation in a dynamically changing set of available channels. The proposed algorithm has a complexity that increases linearly with both the number of channels and the number of users.
\end{abstract}

\section{INTRODUCTION}

Cognitive radio (CR) is an enabling technique for nextgeneration mobile communications and wireless networking. Spectrum sensing and dynamic spectrum access are the two critical aspects for CR networks. Network protocols in general exhibit some form of implicit cooperation, which leads to fairness and good performance for the entire network. Dynamic spectrum access of the secondary users (SUs) can be formulated as a constraint optimization problem, which can be solved by using water-filling or game theory.

A CR network can be regarded as a chaotic game, trying to find the equilibrium of conflicting actions that meet the node's operational goals across different network layers for a specific environment. From game theory, if the CRs do not cooperate, there is a unique Nash equilibrium. Using this solution for CRs, the achievable rate is bounded by a constant, regardless of the available transmit power [8]. In a cooperative spectrum sensing strategy, all CRs collaborate by sharing their decisions regarding spectrum occupancy of the primary users (PUs). Cooperative detection among the CRs can be exploited to relax the requirements on the detectors of the individual CRs or to improve the agility of the entire network.

Some non-cooperative game-theoretic models for dynamic spectrum access have been investigated in the literature. Examples are spectrum load smoothing (SLS) [2] and spectrum load balancing (SLB) [4], and price-based iterative water-filling (PIWF) algorithm [11]. Pricing and resource allocation are closely related, as a service provider wants to maximize its revenue and the user desires to maximize its satisfaction in terms of QoS performance and price [1], [10]. In [13], stochastic learning based techniques are exploited to discover the equilibrium solution.

Cooperation in wireless networks can improve the performance by exploiting some form of multiuser diversity. In a cooperative game, players cooperate by forming a coalition, described by a single payoff. The share of the payoff received by all players in the coalition is a payoff vector. In [9], distributed adaptive channel allocation is modeled to a potential game, which targets at a BER or an equivalent SIR requirement. The no-regret learning algorithm is applied with cooperation on the potential game.

When multiple CRs access (or overlay on) the same channel, one needs to minimize the total power consumption for all the users while satisfying the data rate (or SINR) requirement of each user. A simple iterative distributed power control algorithm that achieves the optimal power allocation is given in [7]. In [5], a near optimal scheme with linear complexity is given for jointly allocating channels and power levels among CRs. The proposed iterative water-filling scheme maximizes the sum capacity subject to individual constraints on users' power budgets. In this paper, we give a more general formulation for the dynamic spectrum access problem targeting at maximizing the sum capacity while satisfying the power budgets of individual CRs as well as the SINR constraints on both CRs and PUs. An iterative waterfilling algorithm is derived, and its performance is examined by simulation.

\section{Formulation of Dynamic Spectrum Access}

Given $N$ available channels and $K$ contending CRs. We intend to maximize the sum capacity of a CR network while each CR has the constraints on BER, power, and channel usage

$$
\max \sum_{k=1}^{K} C_{k}=\sum_{k=1}^{K} \sum_{n=1}^{N} c_{n, k} B \log _{2}\left(1+P_{n, k} \bar{g}_{n, k}\right)
$$


s.t.

$$
\begin{gathered}
\sum_{k=1}^{K} c_{n, k}=1, \quad \forall n \\
\sum_{n=1}^{N} P_{n, k} \leq P_{k, \max }, \quad \forall n \\
P_{n, k} \geq 0, \quad \forall n, k
\end{gathered}
$$

where $n$ runs from 1 to $N, k$ runs from 1 to $K, c_{n, k} \in\{0,1\}$ indicates the assignment of channel $n$ to $\mathrm{CR} k, g_{n, k}$ is the channel power gain on the $n$th channel for CR $k$, $\bar{g}_{n, k}=\frac{g_{n, k}}{N_{0} B}, N_{0}$ is the one-sided noise power spectral density, $B$ the bandwidth of each channel, and $P_{k, \max }$ is the maximum power of the $k$ th CR. Equation (2) denotes that each channel can only be assigned to one CR, and (3) specifies the maximum power of each CR. In (1), $C$ can be replaced by its normalized version $\bar{C}=C / B$.

Since each channel admits only one CR, the SINR of each CR $k$ on channel $n$ should be constrained by a threshold

$$
\gamma_{n, k}=\bar{g}_{n, k} P_{n, k} \geq \gamma_{t h, k}
$$

It can be written as

$$
P_{n, k} \geq \frac{\gamma_{t h, k}}{\bar{g}_{n, k}}=P_{n, k, \min }
$$

In a conservative design, the system allows one or more CRs to coexist with a PU but the received SINR constraint at each PU must be ensured. If the base station for the PUs transmits with power $P_{T}$ at a distance of $d_{B, i}$ from the $i$ th PU and there is one CR on the same channel $n$, the received SINR at the $i$ th PU should be above a threshold [5]

$$
\gamma_{P U, i}=\frac{P_{T} d_{B, i}^{-r}}{P_{n, k} d_{k, i}^{-r}+N_{0}^{\prime} B} \geq \gamma_{0}
$$

where $P_{n, k}$ is the transmit power of the $k$ th CR on channel $n, d_{k, i}$ is the distance between the $k$ th CR and the $i$ th PU, $r$ is the exponent of propagation loss. This constraint is equivalent to

$$
P_{n, k} \leq \frac{P_{T}}{\gamma_{0}}\left(\frac{d_{B, i}}{d_{k, i}}\right)^{-r}-\frac{N_{0}^{\prime} B}{d_{k, i}^{-r}}=P_{n, k, \max }
$$

where $N_{0}^{\prime} B$ is the noise power of a PU.

\section{Solving Using Karush-Kuhn-Tucker Theorem}

The above problem is a mixed-integer nonlinear programming problem. The discreteness of $c_{n, k}$ endows the problem with analytical and algorithmic intractabilities. In order to solve it using nonlinear programming, one can convert the discrete variable $c_{n, k} \in\{0,1\}$ into a continuous variable. In [5], $c_{n, k}$ is treated as a continuous variable in the interval $[0,1]$, and quantize the final result to 0 or 1 . This result is suboptimal, since $c_{n, k}$ 's usually stay in the middle way of the interval. Inspired the fuzzy clustering algorithms where each of the $N$ channels has a membership of each of the $K$ CRs [6], we can change $c_{n, k}$ in (1) to $c_{n, k}^{m}$, where $m \geq 1$ is a fuzzifier. By selecting a large $m$, the optimization process will force one of $c_{n, k}, k=1, \ldots, K$, to approach unity, while all the other $c_{n, k}$ 's to approach zero, since otherwise $c_{n, k}^{m}$ 's will be very small for all $k$.

With PU protection, the problem can be written as

$$
c_{n, k}, P_{n, k}=\arg \max \sum_{k=1}^{K} \sum_{n=1}^{N}\left(c_{n, k}\right)^{m} \log _{2}\left(1+P_{n, k} \bar{g}_{n, k}\right)
$$

s.t.

$$
\begin{gathered}
\sum_{k=1}^{K} c_{n, k}=1, \quad \forall n \\
c_{n, k}\left(1-c_{n, k}\right)=0, \quad \forall n, k \\
\sum_{n=1}^{N} P_{n, k} \leq P_{k, \max }, \quad \forall n \\
P_{n, k} \geq c_{n, k} P_{n, k, \min }, \quad \forall n, k \\
P_{n, k} \leq c_{n, k} P_{n, k, \max }, \quad \forall n, k
\end{gathered}
$$

where $c_{n, k}$ in (13) identifies that there is no SINR constraint for $P_{n, k}$ if the $n$th channel is not assigned to CR $k$.

This is constrainted convex optimization problem. All the constraints are linear. We now solve this problem using the Karush-Kuhn-Tucker (KKT) theorem [3]. By defining an Lagrangian $L\left(P_{n, k}, c_{n, k}, \lambda_{k}, \beta_{n}, \mu_{n, k}\right)$, where $\lambda_{k}, \beta_{n}, \mu_{n, k}$ are multipliers for each of the constraints, the two major KKT conditions are derived by setting the derivatives of $L$ w.r.t. $P_{n, k}$ and $c_{n, k}$, respectively,

$$
\begin{gathered}
\left(c_{n, k}\right)^{m} \frac{1}{\ln 2} \frac{\bar{g}_{n, k}}{\left(1+\bar{g}_{n, k} P_{n, k}\right)}-\lambda_{k}+\mu_{n, k}-\mu_{n, k}^{\prime}=0 \\
m\left(c_{n, k}\right)^{m-1} \log _{2}\left(1+\bar{g}_{n, k} P_{n, k}\right)+\beta_{n}+\beta_{n, k}^{\prime}\left(1-2 c_{n, k}\right) \\
-\mu_{n, k} P_{n, k, \min }+\mu_{n, k}^{\prime} P_{n, k, \max }=0
\end{gathered}
$$

and

$$
\mu_{n, k} \geq 0, \quad \mu_{n, k}^{\prime} \geq 0, \quad \lambda_{k} \geq 0 .
$$

We can set the corresponding $\lambda_{k}^{*}, \mu_{n, k}^{*}$, or $\mu_{n, k}^{\prime *}$ to zero, if an inequality constraint takes on the form of $g_{i}\left(\mathbf{x}^{*}\right)>0$; or set $\lambda_{k}^{*}, \mu_{n, k}^{*}$, or $\mu_{n, k}^{\prime *}>0$ for $g_{i}\left(\mathbf{x}^{*}\right)=0$.

A feasible point that satisfies all the KKT conditions is only a candidate for a maximizer, since the KKT conditions are only necessary conditions. If the Hessian of $L$ is negative definite at the feasible point, it is the optimum solution.

Proposition 1: The Hessian of the Lagrange $L$ is negative semi-definite at the feasible point $\left(P_{n, k}^{*}, c_{n, k}^{*}, \lambda_{k}^{*}\right.$, $\left.\beta_{n}^{*}, \mu_{n, k}^{*}, \mu_{n, k}^{\prime *}\right)$.

The proof is simple. By fixing $c_{n, k}$ in $C$ as unity and then try to maximize $C$ as the function of $P_{n, k}$, the second-order 
derivative is negative. Thus, given $c_{n, k}=1, C$ is convex w.r.t. $P_{n, k}$. As $c_{n, k}$ takes either 0 or 1 , if $c_{n, k}=0, P_{n, k}=0$, thus, $C$ is convex at $c_{n, k}=1$ w.r.t. $P_{n, k}$.

From (15), we can derive $P_{n, k}$. Here we take $m$ as a large number, from which if we want to increase the power of $P_{n, k},\left(c_{n, k}\right)^{m}$ should be as large as possible. In the limit $m, c_{n, k}$ must take unity, in order to increase the power $P_{n, k}$. This is also reasonable, since each channel is assigned to one user. Thus, a water-filling solution is obtained as

$$
\begin{gathered}
P_{n, k}=\left(c_{n, k}\right)^{m} \frac{1}{\lambda_{k}^{\prime} \ln 2}-\frac{1}{\bar{g}_{n, k}} \\
= \begin{cases}P_{n, k}^{0}=\frac{1}{\lambda_{k}^{\prime} \ln 2}-\frac{1}{\bar{g}_{n, k}}, & \text { if } \lambda_{k}^{\prime}<\frac{\bar{g}_{n, k}}{\ln 2}, c_{n, k}=1 \\
0, & \text { otherwise }\end{cases}
\end{gathered}
$$

where

$$
\lambda_{k}^{\prime}=\lambda_{k}-\mu_{n, k}+\mu_{n, k}^{\prime}
$$

If (13) is satisfied at the inequality constraint, then $\mu_{n, k}^{*}=0$. Since $\beta_{n} \geq 0$ and $\mu_{n, k}^{\prime} \geq 0$, from (16), $c_{n, k}=0$. Otherwise, $c_{n, k}=1$ for $k$, which corresponds to $\max _{k} \bar{g}_{n, k} P_{n, k}, k=1,2, \ldots, K$, in order to maximize the channel capacity, since each channel $n$ is assigned to only one user.

In order to achieve the maximum channel capacity, the constraint (12) with equality should be satisfied. Since there are too many parameters to solve from the KKT conditions, it is difficult to solve for the optimum solution. A common strategy is called iterative water-filling.

Substituting (18) into (12), and assuming $\lambda_{k}$ is constant relative to $n$ (that is, assuming $\mu_{n, k}-\mu_{n, k}^{\prime}=$ constant relative to $n$ ), we have

$$
\lambda_{k}^{\prime}=\frac{\left|\mathcal{N}_{k}\right| \frac{1}{\ln 2}}{P_{k, \max }+\sum_{n \in \mathcal{N}_{k}} \frac{1}{\bar{g}_{n, k}}}
$$

where $|\cdot|$ denotes the cardinal of the set within, $\mathcal{N}_{k}$ is the set of channels that are assigned to user $k$, that is, $c_{n, k}=1$, $\forall n \in \mathcal{N}_{k}$.

We now consider the power constraints (13) and (14). We obtain the estimated power by (18), and then verify whether the estimated power satisfies the power constraints. For $c_{n, k}=1$, we have

$$
P_{n, k}=\max \left(\min \left(P_{n, k}^{0}, P_{n, k, \max }\right), P_{n, k, \min }\right)
$$

Accordingly, $\lambda_{k}^{\prime}$ can be updated by replacing $P_{k, \max }$ in the denominator of (20) by $\min \left(P_{k, \max }, \sum_{n \in\left|\mathcal{N}_{k}\right|} P_{n, k, \max }\right)$.

For implementation of iterative water-filling, a centralized agent is necessary. The centralized agent collects all the channel gains $g_{n, k}$ 's, calculates the spectrum assignment, and then broadcasts the channel allocation to the CRs. The algorithm assigns each channel to a user with a maximal capacity for that channel, while subject to the power constraints. Given a channel, if the power constraints cannot be satisfied for any of the users, the channel is not assigned. When applying the power constraints for each user, the unused power budget is assigned the next channel. Implementation of power and channel allocation can be given by the following algorithm.

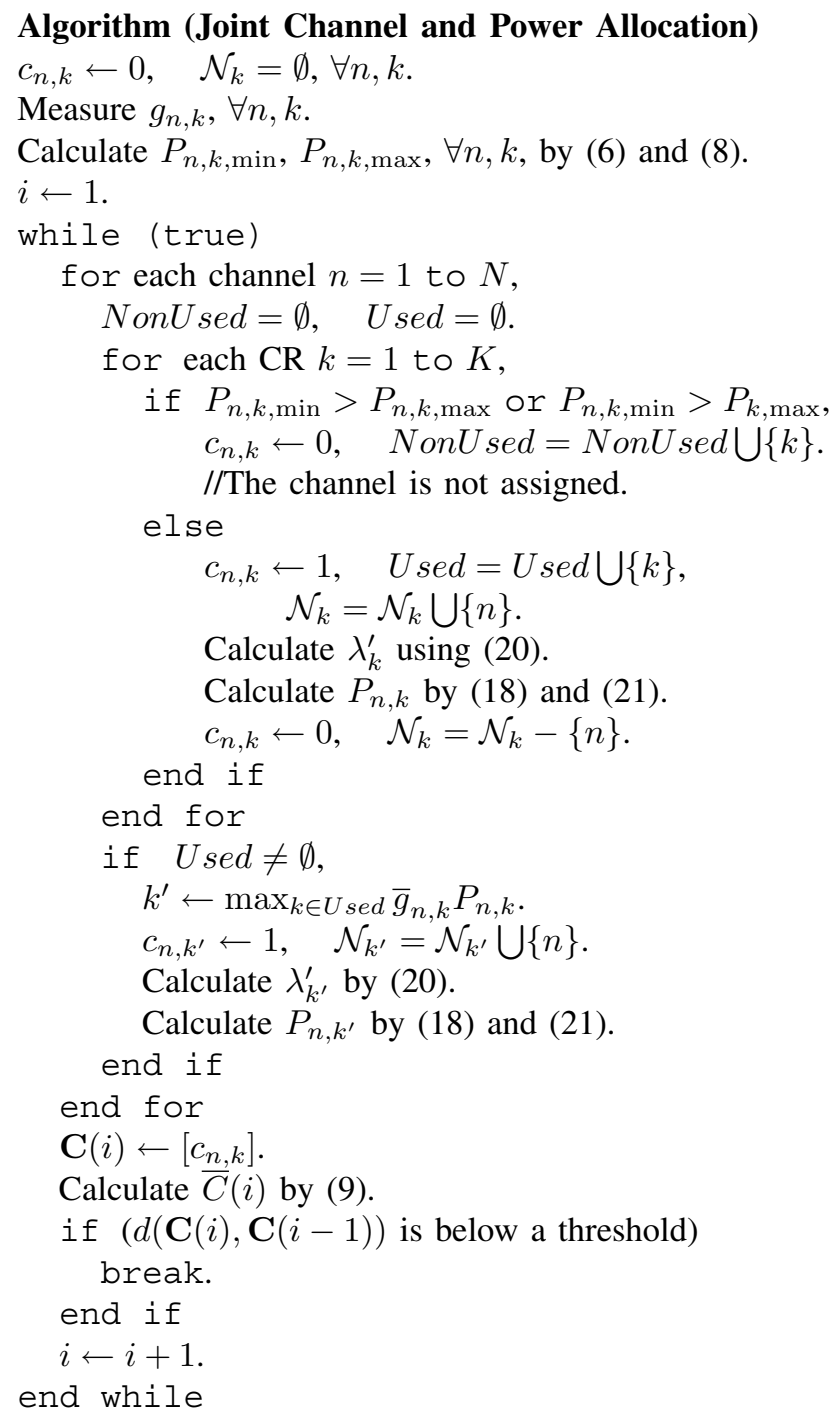

In the algorithm, the distance $d(\mathbf{C}(i), \mathbf{C}(i-1))$ is the Hamming distance between the two matrices, i.e., the number of different entries. The complexity of the algorithm is $O(N K m)$, where $m$ is the number of iterations. This algorithm is of general-purpose nature, and additional constrainsts can be inserted into algorithm.

\section{NumericAl Results}

We now give an example to demonstrate the performance of the iterative water-filling algorithm. All the CRs are assumed to have the same maximum power $P_{k, \max }=1$ watt, $i=1, \ldots, K$. Set the SINR threshod for a CR $\gamma_{t h, k}=5 \mathrm{~dB}$, the noise power at a $\mathrm{CR} N_{0} B=0.1 \mathrm{dBm}$. For the constraint 
(8), the transmit power at the BS of the PU network $P_{T}=5$ watts, the noise power at a PU $N_{0}^{\prime} B=0.1 \mathrm{dBm}$, the distance from the $i$ th PU to its base station $d_{B, i}=12 \mathrm{~m}$, the distance from the $k$ th CR to the $i$ th PU $d_{k, i}=4 \mathrm{~m}$, the propagation exponent $r=2.5$, and the SINR threshold for the PU $\gamma_{0}=5$ $\mathrm{dB}$.

To begin with, we assume that $N=8$ channels are assigned to $K=3 \mathrm{CRs}$. Assume that the gain vectors $\mathbf{g}_{n}$, $n=1, \ldots, N$, are generated by $\mathbf{g}_{n}=(n / N)^{3} \mathbf{a}$, a being a random vector with entries uniformly distributed in $(0,1)$. For a random run, we have

$$
\left[g_{n, k}\right]=\left[\begin{array}{ccc}
0.0011 & 0.0010 & 0.0005 \\
0.0053 & 0.0098 & 0.0022 \\
0.0191 & 0.0356 & 0.0045 \\
0.1248 & 0.0065 & 0.0822 \\
0.1521 & 0.1488 & 0.0809 \\
0.2364 & 0.0996 & 0.3938 \\
0.0808 & 0.5909 & 0.5788 \\
0.3151 & 0.4964 & 0.9788
\end{array}\right]
$$

The algorithm generates the channel assignment $\mathbf{C}=\left[c_{n, k}\right]$ and the corresponding power allocation $\mathbf{P}$ as

$$
\left[c_{n, k}\right]=\left[\begin{array}{lll}
0 & 0 & 0 \\
0 & 1 & 0 \\
0 & 1 & 0 \\
1 & 0 & 0 \\
1 & 0 & 0 \\
0 & 0 & 1 \\
0 & 1 & 0 \\
0 & 0 & 1
\end{array}\right], \quad \mathbf{P}=\left[\begin{array}{rrrr}
0 & 0 & 0 \\
0 & 0.0721 & 0 \\
0 & 0.0779 & 0 \\
0.0779 & 0 & 0 \\
0.0779 & 0 & 0 \\
0 & 0 & 0.0779 \\
0 & 0.0779 & 0 \\
0 & 0 & 0.0779
\end{array}\right]
$$

and $P_{n, k, \max }=0.0779$. The sum capacity is 48.0704 bits/s/Hz. If the constraint for conservative design (i.e., the SINR constraint on the PU) is not applied, $P_{n, k, \max }=$ $P_{k, \max }=1$,

$$
\left[c_{n, k}\right]=\left[\begin{array}{lll}
1 & 0 & 0 \\
0 & 1 & 0 \\
0 & 1 & 0 \\
1 & 0 & 0 \\
1 & 0 & 0 \\
0 & 0 & 1 \\
0 & 1 & 0 \\
0 & 0 & 1
\end{array}\right], \quad \mathbf{P}=\left[\begin{array}{rrrr}
0.2815 & 0 & 0 \\
0 & 0.3275 & 0 \\
0 & 0.3349 & 0 \\
0.3627 & 0 & 0 \\
0.3628 & 0 & 0 \\
0 & 0 & 0.4999 \\
0 & 0.3376 & 0 \\
0 & 0 & 0.5001
\end{array}\right]
$$

and the sum capacity is $66.1165 \mathrm{bits} / \mathrm{s} / \mathrm{Hz}$.

The convergence of the iterative water-filling algorithm is very fast, usually needs 2 to 4 iterations for the algorithm to converge, when $N$ and $K$ are less than 200. Figure 1 shows the convergence of the algorithm for 20 iterations in the case of conservative design with $N=1000$ and $K=500$.

For different values of $N$ and $K$ as well as conservative and nonconservative designs, the sum capacity $\bar{C}$ is plotted in Fig. 2. For this example, the conservative design substantially reduces the sum capacity. For different $N$ and $K$
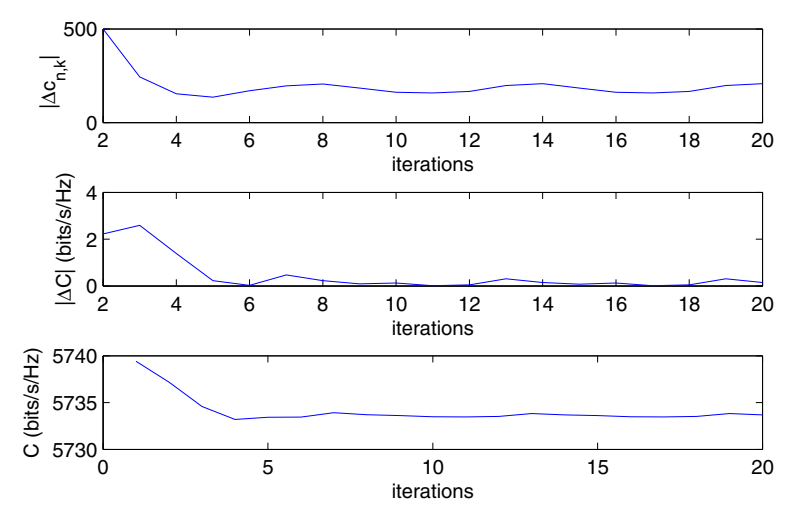

Fig. 1. The evolution of $d(\mathbf{C}(i), \mathbf{C}(i-1)),|\bar{C}(i)-\bar{C}(i-1)|$, and the sum capacity $\bar{C}(i): N=1000, K=500$, and conservative design.

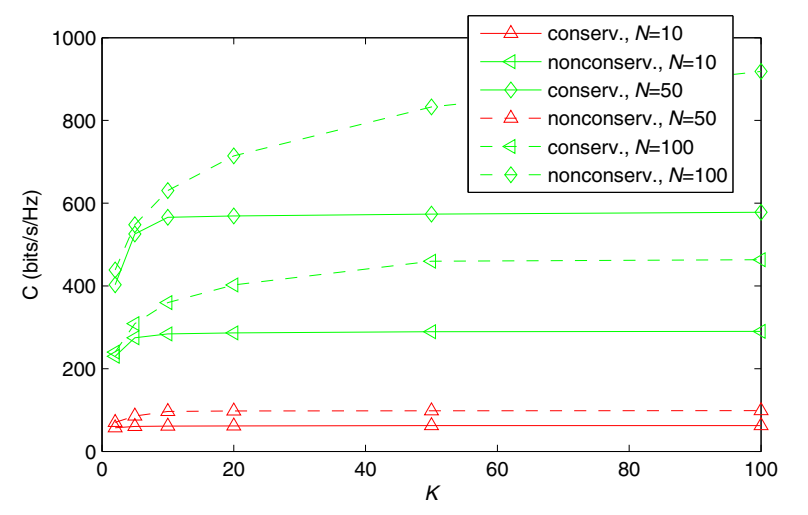

Fig. 2. The sum capacity $\bar{C}$ for different values of $N$ and $K$, with conservative design

values, the sum capacity versus $P_{k, \max }$ is plotted in Fig. 3 . It is shown that when $P_{k, \max }>P_{n, k, \max }$, the sum capacity $\bar{C}$ tends to saturate rapidly for conservative design, while it increases in the logarithmic rate for nonconservative design.

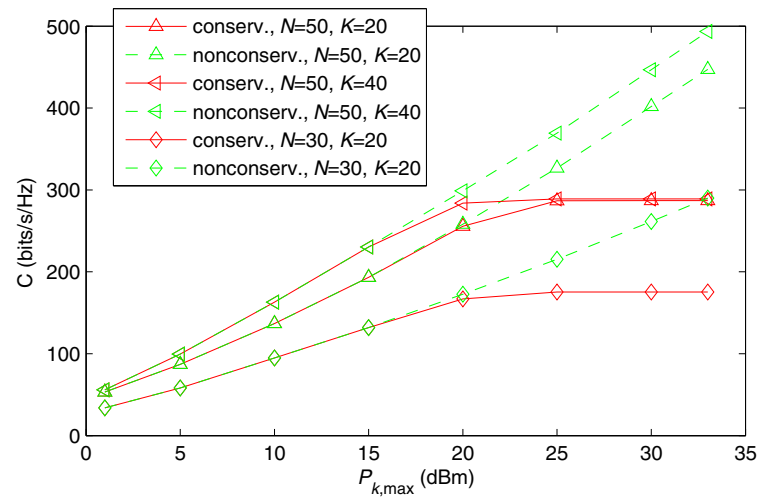

Fig. 3. The sum capacity $\bar{C}$ as a function of $P_{k, \max }$ 


\section{CONCLUSion}

We have addressed the dynamic spectrum access problem for CR networks by maximizing the sum capacity subject to individual $\mathrm{CR}$ power budgets and the receive SINR (or BER) constraints. An iterative water-filling algorithm is proposed that can converge to the final channel and power allocation within a few iterations. The algorithm is very fast, with a complexity that is linear with both the number of channels $N$ and the number of users $K$. Simulation results demonstrate there is a substantial capacity gap between the nonconservative and conservative designs.

\section{ACKNOWLEDGMENT}

K.-L. Du was supported in part by the Hong Kong Research Grants Councils under project number 617706 awarded to Wai Ho Mow of the Hong Kong University of Science and Technology, and in part by the NSERC of Canada grant awarded to M. N. S. Swamy. Qiang Ni was supported by Brunel University's BRIEF award.

\section{REFERENCES}

[1] L. Badia, S. Merlin, A. Zanella, and M. Zorzi, "Pricing VoWLAN services through a micro-economic framework," IEEE Wireless Commun., 13(1), 2006, 6-13.

[2] L. Berlemann and B. Walke, "Spectrum load smoothing for optimized spectrum utilization: rationale and algorithm," Proc. IEEE WCNC, vol. 2, New Orleons, LA, Mar. 2005, 735-740.

[3] E. K. P. Chong and S. H. Zak, An Introduction to Optimization, Wiley, New York, 1996.

[4] A. T. Chronopoulos, M.R. Musku, S. Penmatsa, and D.C. Popescu, "Spectrum load balancing for medium access in cognitive radio systems," IEEE Commun. Lett., 12(5), 2008, 353-355.

[5] F. F. Digham, "Joint power and channel allocation for cognitive radios," Proc. IEEE WCNC, Las Vegas, NV, Mar.-Apr. 2008, 882887.

[6] K. -L. Du and M. N. S. Swamy, Neural Networks in a Softcomputing Framework, Springer, London, 2006.

[7] G. J. Foschini and Z. Miljanic, "A simple distributed autonomous power control algorithm and its convergence," IEEE Trans. Veh. Tech., 42(4), 1993, 641-646.

[8] E. G. Larsson and E. A. Jorswieck, "The MISO interference channel: competition versus collaboration", Proc. Allerton Conf. Commun., Control, and Computing, Monticello, AR, Sep. 2007.

[9] N. Nie and C. Comaniciu, "Adaptive channel allocation spectrum etiquette for cognitive radio networks", Proc. IEEE DySPAN, Baltimore, MD, Nov 2005, 269-278.

[10] D. Niyato and E. Hossain, "Competitive pricing for spectrum sharing in cognitive radio networks: dynamic game, inefficiency of Nash equilibrium, and collusion,” IEEE JSAC, 26(1), 2008, 192-202.

[11] F. Wang, M. Krunz, and S. Cui, "Price-based spectrum management in cognitive radio networks," IEEE J Sel. Topics Signal Process., 2(1), 2008, 74-87.

[12] Y. Xing, R. Chandramouli, S. Mangold, and S. Shankar N, "Dynamic spectrum access in open spectrum wireless networks," IEEE JSAC, 24(3), 2006, 626-637.

[13] Y. Xing, H. Kushwaha, K. P. Subbalakshmi, and R. Chandramouli, "Codes and games for dynamic spectrum access," in H. Arslan (Ed.), Cognitive Radio, Software Defined Radio, and Adaptive Wireless Systems, Springer, Berlin, 2007, 161-187. 